\title{
NANO-PARTICLE SEPARATION USING MARANGONI FLOW IN EVAPORATING DROPLETS Hwapyeong Jeong ${ }^{1,2}$, Joël van Tiem ${ }^{1,3}$, Yogesh B. Gianchandani ${ }^{1}$ and Jaesung Park ${ }^{1,2}$ \\ ${ }^{1}$ Center for Wireless Integrated MicroSensing and System (WIMS ${ }^{2}$ ), U. Michigan, Ann Arbor, MI 48109, USA \\ ${ }^{2}$ Pohang University of Science and Technology, Republic of Korea \\ ${ }^{3}$ University of Twente, The Netherlands
}

\begin{abstract}
The "coffee ring effect" is known to result from the concentration of particles at the perimeter of a dried droplet. Raising the temperature of the substrate provides a temperature gradient induces "strong” Marangoni circulatory flow inside the droplet. This "strong" Marangoni can reverse the "coffee ring" effect. Here, we describe a method of particle separation based on the size of particles using Marangoni flow and the related "coffee ring” effect. Suspended particles of different sizes ranging from $100 \mathrm{~nm}$ to $15 \mu \mathrm{m}$ are separated by using a combination of the "strong" Marangoni flow and coffee ring effects. Additionally, the effects of droplet contact angle with the substrates and temperature gradient in droplets are evaluated. Experiments show that, a temperature gradient of $1^{\circ} \mathrm{C} / \mathrm{mm}$, and contact angle $\left(15^{\circ}\right)$ can separate $100 \mathrm{~nm}$-particles from $1 \mu \mathrm{m}$-particles successfully. The distance between the dried ring patterns is $\sim 30 \mu \mathrm{m}$. In contrast, the separation between rings of

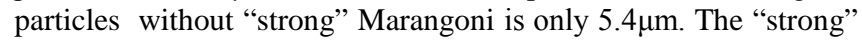
Marangoni flow induced by temperature gradient enhances the separation of nano-particles at the contact line of a droplet.
\end{abstract}

\section{INTRODUCTION}

The "coffee ring effect" is a commonly observed upon evaporating liquid droplets containing colloidal particles. A coffee droplet leaves a ring pattern behind from the coffee droplet as it dries in the absence of a temperature gradient. Deegan et al.[1] were the first to propose the physical model which showed that the main cause of "coffee ring" effect is the outward capillary flow within evaporating liquid droplet with a pinned contact line. Here, liquid evaporating from the edge is replenished by liquid from the interior. Later, Hua et al. showed that a "weak" Marangoni flow is also induced by surface tension gradients associated with the latent heat of evaporation, and reverses "coffee ring" deposition [2].

However, in the case that circulating flow ("weak" Marangoni flow) is induced by capillary effect of the drying contact line, particles inside the evaporating liquid droplets can be easily free from this unclear weak capillary flow due to small buoyance from smaller temperature gradient from latent heat, and cannot contribute to ring formation.

Unlike “weak” Marangoni flow, “strong” Marangoni circulatory flow is developed inside the droplet by raising temperature of the substrate (Fig. 1). In this case, colloidal particles in the droplet move faster in the overall circulatory flow, because larger buoyancy is developed by the applied temperature gradient. Additionally, this larger buoyancy makes more particles move along the circulatory flow. This "strong" Marangoni flow can carry more particles at faster speed.

Recently, methods utilizing evaporating liquid droplets have drawn attention for various applications, such as particle self-assembly, film coating and particle separation and concentration for spot test analysis [3-6]. Most of the studies use evaporating droplets without an applied temperature gradient, and focus on "coffee ring". In our previous work, we reported that "strong” Marangoni flow deposit larger micro-particles at the center of droplets, and small micro-particles at the contact line of the droplet in size-orderly fashion [7].
This paper describes the use of droplet drying as a method for separating $100 \mathrm{~nm}$-particles from 500nm-particles that are in aqueous solution. Substrates with different contact angles for the droplets are tested, and the shallowest contact angle, $15^{\circ}$ shows separated "coffee ring" pattern. For enhancing the spatial resolution of the pattern, "strong” Marangoni flow is used. The results show that hydrophilic substrates with $15^{\circ}$ contact angle can separate 100nm-particles, and "strong" Marangoni flow improves the resolution about 6 times. The proposed conditions can be useful for improving chromatography and disease diagnosis in health care fields.

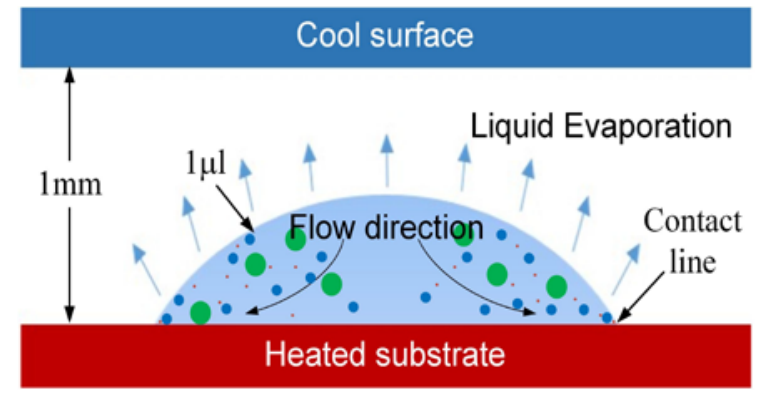

Figure 1: A scheme of concept of particle separation system with temperature gradient. The liquid in the droplet evaporates on the free surface and causes "weak" Marangoni flow while the temperature gradient imposed by the hot and cold surface causes "strong" Marangoni flow.

\section{THEORY AND SIMULATION}

The presence of a surface-tension gradient on a liquid droplet creates circulatory flow in the droplet. A surface-tension gradient can be induced by either latent heat of evaporation or a temperature gradient forced by boundary conditions (Fig. 1). These gradients create Marangoni flow. In Marangoni flow, the thermo-capillary shear stress is proportional to the temperature gradient [7]:

$\tau_{S}=\mu \frac{\partial v_{S}}{\partial \hat{n}}=-\sigma_{T} \nabla T_{S}$

where $\mu$ is the dynamic viscosity of the liquid, $\tau_{s}$ denotes the surface stress, $v_{s}$ is the surface velocity, $T$ is the temperature, $\hat{n}$ and $\hat{t}$ are the unit normal and tangential vector to the surface, respectively, and $\sigma_{T}$ is the surface tension temperature coefficient. Marangoni flow is generated inside the liquid droplet. (The surface tension increases with a decreasing temperature due to negative value of $\sigma_{T}$ for common fluids) As a result of surface tension gradient induced by temperature gradient, flow moves toward to colder regions at the liquid interface. It causes viscous drag, which moves fluid, establishing a convective Marangoni flow. When a temperature gradient is applied to a liquid droplet, usually parasitic effect, buoyancy effect are present at the same time. The relative strengths of the Marangoni effect and buoyancy are estimated by the dimensionless numbers, Marangoni number (Ma) and Rayleigh number $(\mathrm{Ra})$ [8]. 


$$
\begin{aligned}
& M a=\frac{\sigma_{T} \Delta T R}{\mu \kappa} \\
& R a=\frac{\alpha \rho g \Delta T R^{3}}{\mu \kappa}
\end{aligned}
$$

where $\Delta T$ is temperature difference, $R$ is the characteristic length (droplet radius), $\kappa$ is the thermal diffusivity, $\rho$ is the liquid density, $\alpha$ is the liquid thermal expansion coefficient, and $g$ is the gravitational acceleration. The ratio of both numbers provides an indication of the dominance of thermo-capillary force over buoyancy force.

$$
\frac{M a}{R a}=\frac{\sigma_{T}}{\alpha \rho g R^{2}}
$$

The Marangoni effect is dominant over buoyancy force when $M a / R a>>1$. In this research, buoyancy force is negligible because the size of droplet, $1 \mu \mathrm{L}$ is small. However, temperature gradient is imposed by a constant temperature boundary condition (Fig. 1). Accordingly, depending on the situation, buoyancy forces may play important roles.

The flow pattern inside liquid droplets is analyzed using finite element analysis (COMSOL ${ }^{\mathrm{TM}}$ ). The temperature difference and the distance between substrates are $1^{\circ} \mathrm{C}$ and $1 \mathrm{~mm}$, respectively. To confirm the impact of contact angle, various geometries are chosen in such a way that the droplets have the same volume. Marangoni flow results in recirculation and transport of the suspended particles inside the droplets (Fig. 2 a-c). Since the profile of the droplet on the hydrophobic substrate is much higher than on the hydrophilic, the liquid surface is closed to the cold substrate. Therefore, the temperature gradient is higher and the magnitude of velocity increases. However, the velocity outward to the contact line of the droplet is faster on the hydrophilic substrate than on the hydrophobic substrate, indicating that particles near the hydrophilic substrate experience more drag force than those on the hydrophobic substrates. According to simulations, trajectories of $5 \mu \mathrm{m}-$ and $50 \mu \mathrm{m}$-particles are different due to the difference in buoyancy force (Fig 2d). Therefore, the larger particles circulate near the center of the liquid droplet, and the smaller particles move toward to contact line by outward flow along the bottom substrate.

\section{EXPERIMENTS}

The fluorescence polystyrene sphere of $15 \mu \mathrm{m}, 3 \mu \mathrm{m}, 1 \mu \mathrm{m}, 500 \mathrm{~nm}$, $100 \mathrm{~nm}$ in diameter were prepared in deionized water with an initial volume fraction of about $0.003 \%$. The density of these particles is $1.05 \mathrm{~g} / \mathrm{cm}^{3}$. The temperature of the substrate was maintained by using constant temperature blocks connected to a circulating water bath at $35^{\circ} \mathrm{C}$ for cases without an applied temperature gradient. In other cases, the temperatures of the lower hot substrate and upper cool surface were maintained $36^{\circ} \mathrm{C}$ and $35^{\circ} \mathrm{C}$, respectively, by using a hot plate and a circulating water bath, respectively. The distance between the heated and cooled surfaces was $1 \mathrm{~mm}$. The volume of a droplet was $1 \mu \mathrm{L}$.

To confirm the effect of contact angle, samples were placed on various surfaces with different contact angles, such as commercial slide glass, parylene coated glass and polydimethylsiloxane (PDMS). Contact angle of commercial slide glass, PDMS and parylene- coated glass are $\sim 15^{\circ}, \sim 90^{\circ}$ and $\sim 110^{\circ}$, respectively. Then the samples were placed on those substrates and dried with or without temperature gradient. After the droplets on the substrates were dried, "coffee ring” patterns appeared near the contact lines between the droplets and the substrates. The ring patterns were observed by using fluorescence microscope. The experiment environment was maintained under $40 \%$ humidity of ambient air to minimize the effect of evaporation time.

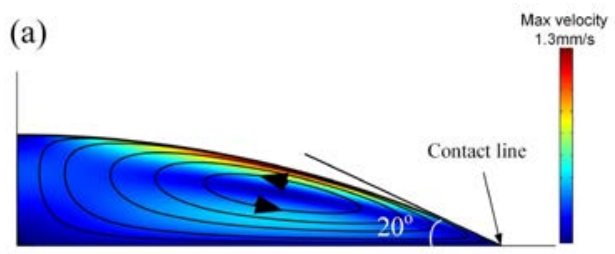

(b) (c)

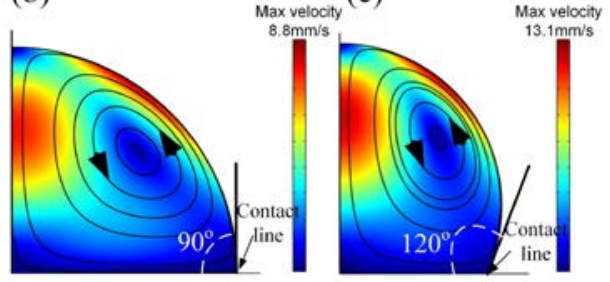

(d)

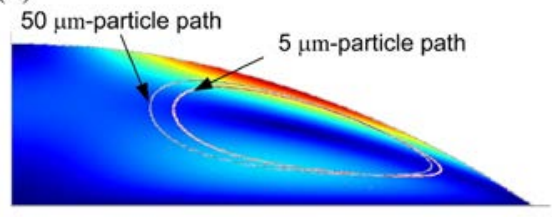

Figure 2. Modeling results in $1 \mu \mathrm{L}$ droplet. (a) flow velocities on hydrophilic substrate with shallow contact angle $\left(20^{\circ}\right)$. (b) flow velocities on hydrophobic substrate $\left(90^{\circ}\right)$. (c) flow velocities on hydrophobic substrate $\left(120^{\circ}\right)$. (d) trajectories of $5 \mu \mathrm{m}$ and $50 \mu \mathrm{m}$ particles.

\section{RESULTS}

To evaluate the effects of temperature and contact angle, 100nmparticles and $1 \mu \mathrm{m}$ particles were mixed and suspended in aqueous droplets $(1 \mu \mathrm{L}$ volume, $0.003 \%$ volume fraction). First, the effect of contact angle was investigated. The glass, PDMS, and parylene-coated glass substrates were compared without temperature gradient. Droplets were deposited on each substrate. On glass (contact angle $\sim 15^{\circ}$ ), the dried ring patterns of $100 \mathrm{~nm}$ - and 1 $\mu \mathrm{m}$-particles became separated by $\sim 5.4 \mu \mathrm{m}$ (Fig. 3 a). This separation was estimated visually under a microscope. On PDMS substrates (contact angle $\sim 90^{\circ}$ ), the dried ring patterns were partially overlapped and not distinguishable, although the dried particle rings showed a gradual color change from green to blue inward (Fig. 3 c). In case of the hydrophobic parylene-coated substrates (contact angle $\sim 110^{\circ}$ ), the dried ring patterns were likely to overlap or oppose to the previous cases. The results indicate that the contact angle between the droplet and substrate is critical in separation of particles in the droplets, and nano-particles can be separated from micro-particles using hydrophilic substrate (contact angle $\sim 15^{\circ}$ ).

To investigate the effect of strong Marangoni flow, with temperature gradient of $1{ }^{\circ} \mathrm{C} / \mathrm{mm}$, the same experiments were repeated on the same substrates. The measured separation between the dried ring particles $(100 \mathrm{~nm}$ and $1 \mu \mathrm{m})$ was measured to be $29.4 \mu \mathrm{m}$ on the glass substrate (contact angle $\sim 15^{\circ}$ ) (Fig. $3 \mathrm{~b}$ ). Compared to the case of "weak" Marangoni flow, the distance of patterns was $6 \mathrm{X}$ larger. On the PDMS substrate (contact angle $90^{\circ}$ ), the dried ring pattern of $100 \mathrm{~nm}$-particles become wider and overlapped with that of $1 \mu \mathrm{m}$-particles (Fig. 3d). On the hydrophobic parylene-coated glass substrate (contact angle $\sim 110^{\circ}$ ), the dried ring patterns of $100 \mathrm{~nm}$ - and $1 \mu \mathrm{m}$-particles were overlapped, and not distinguishable. 

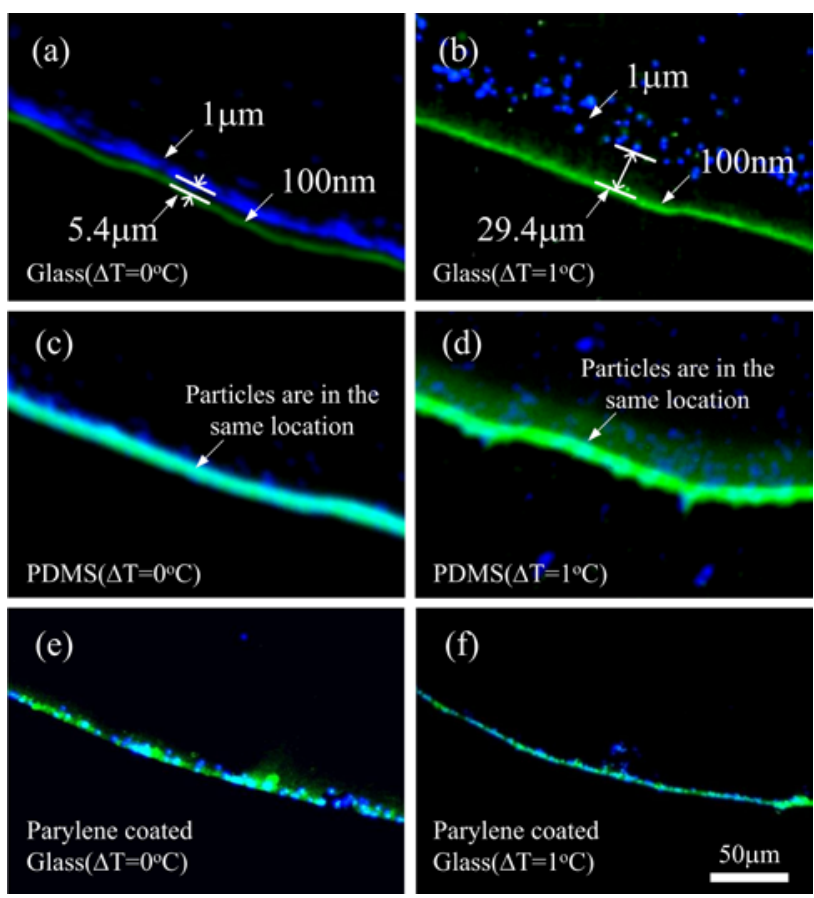

Figure 3. Fluorescence images of the dried patterns separation of $1 \mu \mathrm{m}$ (blue) and 100nm (green)-particles. (a) on hydrophilic glass substrate without an applied temperature gradient. (b) hydrophilic glass substrate with temperature gradient $\left(\Delta T=1^{\circ} \mathrm{C}\right)$. (c) hydrophobic PDMS substrate without an applied temperature gradient. (d) hydrophobic PDMS substrate with temperature gradient. (e) hydrophobic parylene-coated glass substrate without an applied temperature gradient. ( $f$ ) hydrophobic parylene-coated glass substrate with temperature gradient.

When the substrate is hydrophilic, the contact line is pinned because the aqueous droplet tends to adhere to the substrate (Fig. 4a). During evaporation, "weak" Marangoni flow induced by latent heat pushes the particles toward the contact line, and the suspended particles are kinematically pinched between the free surface line of the droplet and substrate (Fig. 4a). As the droplet evaporates, the contact angle becomes smaller and able to pinch particle larger than the previously pinched ones. These explanation match well with experimental results (Fig. 3). Compared to the case with "weak" Marangoni flow, the case with "strong" Marangoni flow shows better separation distance. The reason may be that the "strong" Marangoni flow pushes harder toward the contact line because "strong" Marangoni flow has higher speed than "weak" Marangoni flow. As a result, the distance between the dried ring patterns increases (Fig. 3b).

The distance between successive ring patterns, $\Delta d$, can be simply estimated from the geometry of droplet profile if surface is hydrophilic [9]:

$$
\Delta d=\frac{\Delta D}{2 \tan (\theta / 2)}
$$

where $\Delta D$ is difference of diameter of particles, and $\theta$ is initial contact angle of droplet below $90^{\circ}$. According to this equation, as the contact angle, $\theta$ increases, the separation distance, $\Delta d$ decreases.

For the $100 \mathrm{~nm}$ - and $1 \mu \mathrm{m}$ particles, the distance calculated from Equation (5) is $3.14 \mu \mathrm{m}$ while the measured distance for "weak" Marangoni without temperature gradient was $5.4 \mu \mathrm{m}$ and for "strong" Marangoni with temperature gradient, it was $29.4 \mu \mathrm{m}$ (Fig. 3a, b). (a)

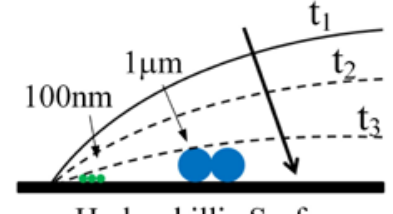

Hydrophillic Surface

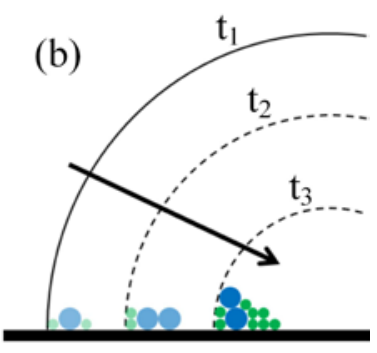

Hydrophobic Surface
Figure 4. Scheme for dynamic motion of suspended particle inside the liquid droplet during evaporation. (a) the line on the hydrophilic substrate does not move due to strong adhesion between the droplet and the substrate, whereas the contact angle changes smaller. (b) the contact angle on the hydrophobic substrate does not change due to weak adhesion between the droplet and the substrate, whereas the contact line move inwardly.

In contrast to the hydrophilic substrate, the contact line on hydrophobic substrate is not pinned because the aqueous droplet does not stick on the substrates as strong as the hydrophilic substrate (Fig. 4b). Unlike the hydrophilic substrate, the contact angle does not change during evaporation. With the large contact angle, particles are not pinched, and are transported away from the contact line by Marangoni flow (Fig 2c). Eventually, the suspended particles do not form distinguishable patterns at the contact line (Fig 3f). As proof of that, the diameters of the dried ring pattern on hydrophobic substrate were smaller than the hydrophilic substrate. (Fig. 4).
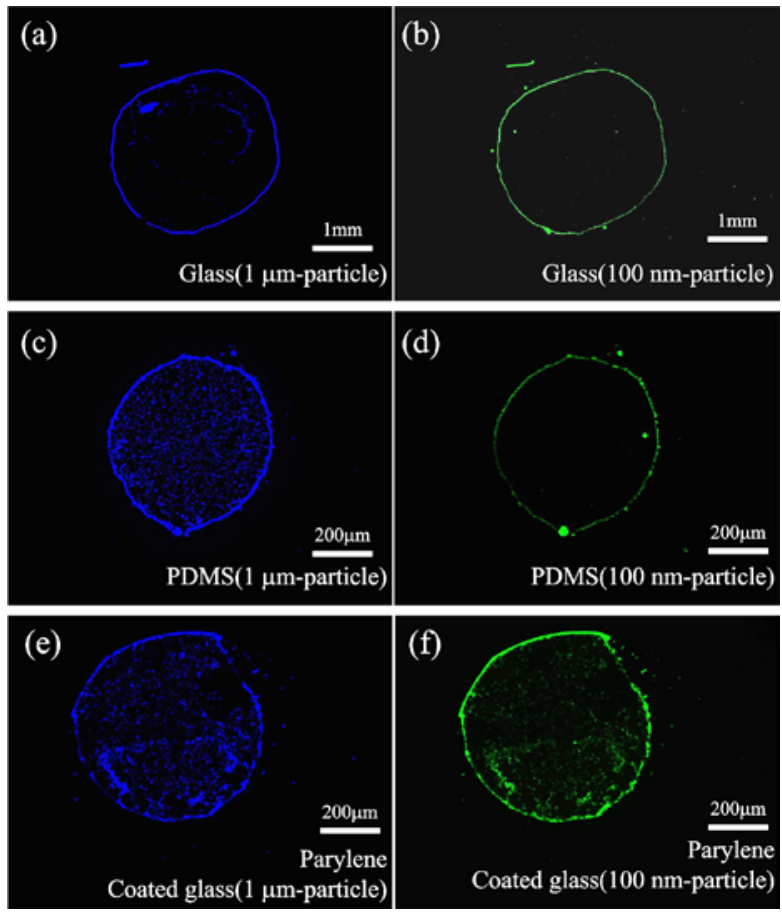

Figure 5. Scheme for dynamic motion of suspended particle inside the liquid droplet during evaporation. (a) on glass substrate (contact angle $\sim 15^{\circ}$ ), the diameter of the dried ring pattern did not shrink. (b),(c) on PDMS (contact angle $\sim 90^{\circ}$ ) and parylene-coated $\left(\sim 110^{\circ}\right)$, the diameters of the dried ring patterns shrunk, compared to the initial diameters. 


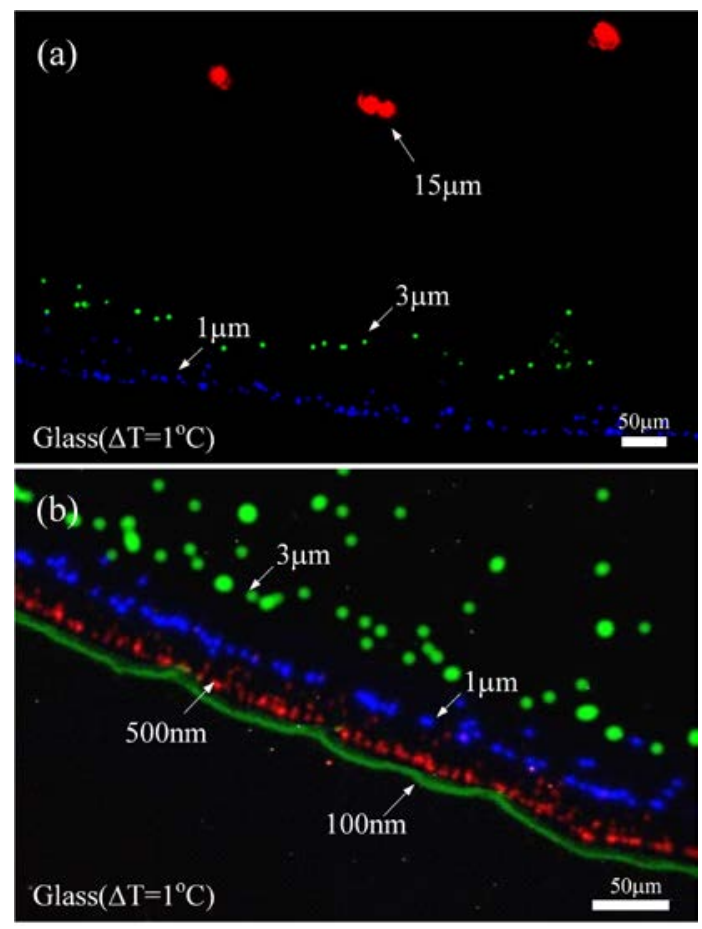

Figure 6. (a) A fluorescence image of the separation of $1 \mu \mathrm{m}$ (blue), $3 \mu \mathrm{m}$ (green), $15 \mu \mathrm{m}$ (red) particles after evaporation on hydrophilic substrate (glass) with temperature gradient. (b) A fluorescence image of the separation of 100nm (green), 500nm (red), $1 \mu \mathrm{m}$ (blue), $3 \mu \mathrm{m}$ (green)- particles after evaporation on hydrophilic substrate (glass) with temperature gradient.

Leveraging the findings described above, experiments were also performed to test separation across a wide range of particle sizes. The experiments were repeated with suspended particles in range of $100 \mathrm{~nm} \sim 15 \mu \mathrm{m}(1 \mu \mathrm{L}$ droplet volume, $0.003 \%$ volume fraction) under the same conditions described previously. For better separation, "strong" Marangoni flow with $1^{\circ} \mathrm{C} / \mathrm{mm}$ was used. First, dried pattern showed that micro-particles, $1 \mu \mathrm{m}, 3 \mu \mathrm{m}$ and $15 \mu \mathrm{m}$, were clearly sorted based on their sizes. Once the smallest particles were deposited at the outermost ring near the contact line, the second and third size patterns were arranged and deposited sequentially by their size, as expected (Fig. 6 a). The 100nm-, 500nm-, $1 \mu \mathrm{m}-$ and $3 \mu \mathrm{m}$-paricles, were also separated clearly (Fig. 5 b). Separation of $100 \mathrm{~nm}$ and $500 \mathrm{~nm}$ was particularly successful. The particles ranging from $100 \mathrm{~nm}$ to $3 \mu \mathrm{m}$ were separated in orderly fashion, and the ring patterns were clearer than the patterns of micro-particles. One possible reason for this difference is larger size tolerance in micro-particles. This result shows that the separation method using Marangoni flow on evaporating liquid droplet can be used to separate and concentrate various sizes of particles simultaneously.

\section{CONCLUSION}

This effort demonstrated nano-particle separation method using Marangoni flow and contact angle. It was found that only hydrophilic substrates can be used to separate suspended particle inside the liquid droplet. On hydrophobic substrates, the suspended particles cannot not be pinned at the contact line and are transported by receding movement during evaporation. The distance between smaller and larger particles is amplified by using "strong" Marangoni flow that is induced by a forced temperature gradient during evaporation. This technique is simple and low-cost, minimizing contamination of samples, and small amount of sample volume. In the future, this method can be extended to diagnostic applications.

\section{ACKNOWLEDGEMENTS}

This work was supported in part by the National Research Foundation of Korea (NRF) grant funded by the Korea government (MSIP) (No. 2011-0030075, 2011-0028845) and in part by the University of Michigan.

\section{CONTACT}

Jeasung Park, tel: +82-54-279-2188; jpark@postech.ac.kr Yogesh B. Gianchanani: +1-734-615-6407;yogesh@umich.edu

\section{REFERENCES}

[1] R. D. Deegan, O. Bakajin, T. F. Dupont, G. Huber, S. R. Nagel, and T. A. Witten, "Capillary flow as the cause of ring stains from dried liquid drops," Nature, vol. 389, pp. 827-829, 1997.

[2] H. Hu and R. G. Larson, "Marangoni effect reverses coffee-ring depositions," The Journal of Physical Chemistry B, vol. 110, pp. 7090-7094, 2006.

[3] J. R. Trantum, D. W. Wright, and F. R. Haselton, "Biomarker-mediated disruption of coffee-ring formation as a low resource diagnostic indicator," Langmuir, vol. 28, pp. 2187-2193, 2011.

[4] J. R. Trantum, M. L. Baglia, Z. E. Eagleton, R. L. Mernaugh, and F. R. Haselton, "Biosensor design based on Marangoni flow in an evaporating drop," Lab on a Chip, vol. 14, pp. 315-324, 2014.

[5] T.-S. Wong, T.-H. Chen, X. Shen, and C.-M. Ho, "Nanochromatography driven by the coffee ring effect," Analytical chemistry, vol. 83, pp. 1871-1873, 2011.

[6] V. Vohra, A. Bolognesi, G. Calzaferri, and C. Botta, "Self-assembled nanofibers of fluorescent zeolite L crystals and conjugated polymer," Langmuir, vol. 26, pp. 1590-1593, 2010.

[7] E. Hendarto and Y. B. Gianchandani, "Size sorting of floating spheres based on Marangoni forces in evaporating droplets," Journal of Micromechanics and Microengineering, vol. 23, p. 075016, 2013.

[8] S. T. Chang and O. D. Velev, "Evaporation-induced particle microseparations inside droplets floating on a chip," Langmuir, vol. 22, pp. 1459-1468, 2006.

[9] X. Shen, C.-M. Ho, and T.-S. Wong, "Minimal size of coffee ring structure," The Journal of Physical Chemistry B, vol. 114, pp. 5269-5274, 2010. 